Anastasia A. Gornostayeva ${ }^{1 *}$, Dmitry Yu. Demezhko

${ }^{1}$ Institute of Geophysics, Ural Branch, Russian Academy of Sciences; 100 Amundsen

Str., Ekaterinburg, Russia; e-mail: free_ride_@mail.ru

*Corresponding author

${ }^{2}$ Institute of Geophysics, Ural Branch, Russian Academy of Sciences; 100 Amundsen

Str., Ekaterinburg, Russia; e-mail: ddem54@inbox.ru

\title{
RECOVERY FROM THE LITTLE ICE AGE: GEOTHERMAL EVIDENCES
}

ABSTRACT. We applied geothermal method for paleoclimatic reconstruction of the ground surface temperature history during the Little Ice Age and contemporary warming. We analyzed 83 borehole temperature profiles and estimated warming amplitudes and warming start dates after the Little Ice Age. The studied boreholes are situated in the Urals and Eastern Europe (Finland, Ukraine, and Belarus). Our investigation shows high degree of spatial variability of climatic changes in 18-19 centuries. Spatial distribution of amplitudes of paleoclimatic changes and warming start date testifies that warming following after the Little Ice Age was in progress in several steps and for different regions it started at different times.

KEYWORDS: paleoclimate, ground surface temperature history (GSTH), Little Ice Age (LIA), Urals, Eastern Europe, borehole temperature-depth profile, geothermal method, spatial distribution of paleoclimatic changes.

\section{INTRODUCTION}

Scientific interest to climatic changes, taking place in the $18^{\text {th }}-20^{\text {th }}$ centuries, is staying constantly high within a few recent decades. The Little Ice Age (LIA) and contemporary warming are the latest significant climatic events on the Earth. Geothermal method for reconstruction of the ground surface temperature history (GSTH) allows estimating past climatic changes occurred during this period [Lachenbruch and Marshall, 1986; Beltrami and Mareshal, 1991; Cermak et al., 1992]. Significant differences of individual borehole GSTHs are linked not so much to non-climatic factors as to spatial-temporal heterogeneities of global climatic changes for many Earth's regions [Majorowicz, 2010; Beltrami et al., 2003]. These heterogeneities may be caused both by natural reasons and non-climatic noise, and may be suppressed by data averaging over a large region [Demezhko, Golovanova, 2007]. In this case, a reliable average estimation of climatic changes for a given territory is appropriate. However, this approach loses a great deal of useful information about spatial paleoclimate features.

In this paper, having a large database of shallow boreholes, we attempted to estimate spatial-temporal features of ground surface temperature and receive useful information which could be lost in the process of GSTH averaging. We compared spatial distribution characteristics of climatic data in the Urals and Eastern Europe (Ukraine, Belarus and Finland) where geothermal reconstructions density is much lower than in the Urals.

\section{MATHEMATICAL MODEL}

Heat transfer in a homogeneous rock medium without vertical ground water flow can be described in terms of a one-dimensional heat equation [Carslaw and Jaeger, 1959]:

$\frac{\partial^{2} T}{\partial z^{2}}-\frac{1}{a} \frac{\partial T}{\partial t}=0$,

where $T$ is temperature, $z$ is depth, $t$ is time and $a$ is thermal diffusivity (thermal diffusivity $a$, thermal conductivity $\lambda$, density $\rho$ and 
specific heat capacity c can be combined in the form $\lambda=\rho(a)$. The solution of Eq. (1) can be expressed as a sum:

$T(z, t)=T_{0}+G_{0} z+\Theta(z, t)$,

where the original surface temperature $T_{0}$ and geothermal gradient $G_{0}$ corresponding to the undisturbed part of the temperature field, and $\Theta$ is a non-stationary temperature anomaly that appears at the moment $t=0$ and satisfies the condition at infinity:

$\Theta(z, t)=0, z \rightarrow \infty$

The surface boundary condition $T_{s}$ can be taken as a surface temperature history approximation in the form of a series of $m$ instantaneous surface temperature changes:

$$
\begin{aligned}
& T_{s}(0, t)= \\
& =\left\{\begin{array}{ll}
T_{0}, & t \leqslant 0 \\
T_{k}, t_{k-1} \leqslant t \leqslant t_{k}, & k=1,2, \ldots, m-1 \\
T_{m}, & t \geqslant t_{m}
\end{array}\right\} .
\end{aligned}
$$

For the conditions given and $t=t^{*}$ (moment of measurement) temperature anomaly has the form:

$\theta\left(z, t^{*}\right)=\sum_{k=1}^{m} D_{k} \operatorname{erfc} \frac{z}{2 \sqrt{a\left(t^{*}-t_{k}\right)}}$,

where erfc $U$ is the complementary error function, $D_{k}=T_{k}-T_{k-1}$ and $t^{*}-t_{k}$ is the time interval from the $k$ th instantaneous temperature change till the moment of measurement. To determine the ground surface temperature history $T_{s}(0, t)$, we 1) evaluated unknown parameters $G_{0}$ and $T_{0}$ from the base interval of the temperature-depth

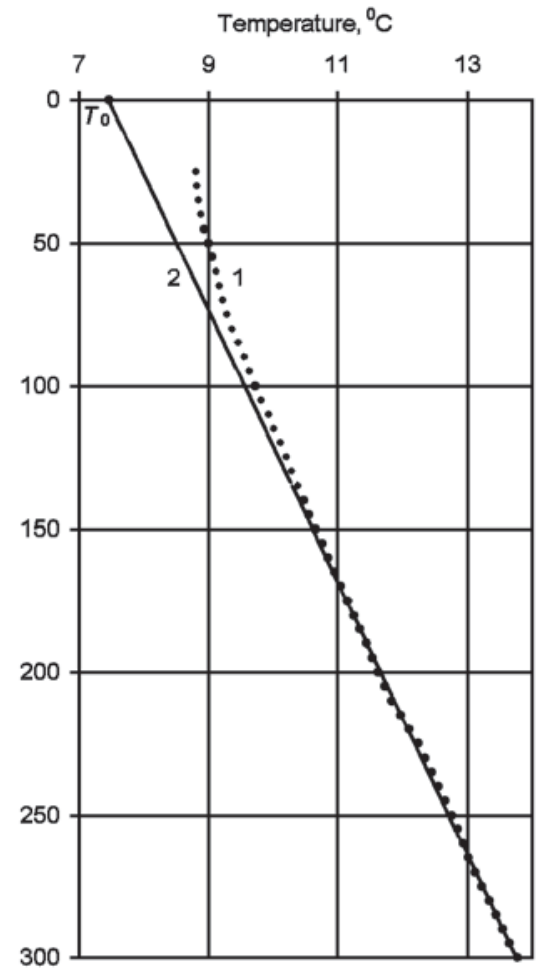

Depth, $m$

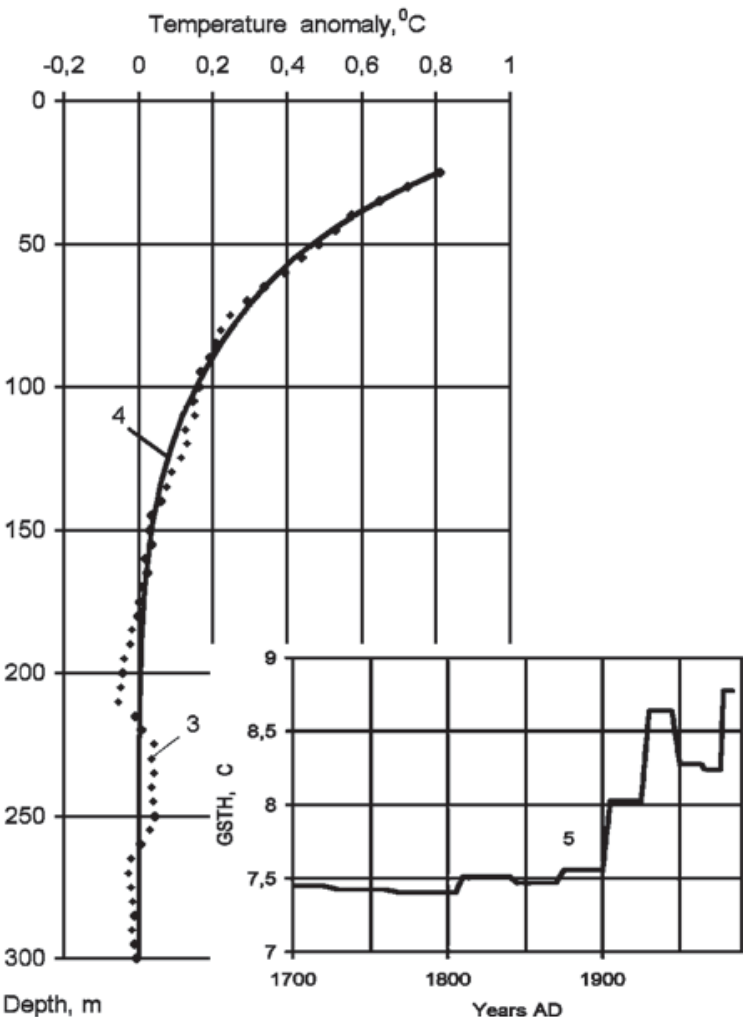

Depth, $m$

Fig. 1. An example of inversion technique utilization:

1 - measured temperature-depth profile GViZD214 (http://www.geo.Isa.umich.edu/climate/);

2 - undisturbed temperatures $T(z)=T 0+G 0 z$ estimated from the deepest part of temperature-depth profile (200-300 m); 3 - temperature anomaly; 4 - calculated temperature anomaly corresponding to GSTH 
profile, 2) estimated temperature anomaly as the difference between measured and undisturbed temperatures, and 3) minimized the mean square error between measured temperature change and calculated anomaly (according to equations 4 and 5) by means of variation of surface temperatures $T_{k}$ in the given time intervals. [D.Yu. Demezhko and V. A. Shchapov, 2001]. An example of inversion technique utilization is shown in Fig. 1.

\section{DATA AND ANALYSES}

We used the temperature-depth profiles recorded in the Urals [Demezhko and
Golovanova, 2007] and European boreholes [Huang and Pollack, 1998] as the initial data for analysis of climatic changes. Location of boreholes is introduced in Fig. 2. It is noteworthy that the density of the temperaturedepth profiles in the Urals is well over the density in the territory of Eastern Europe.

83 temperature profiles at least 300-meters deep were studied. Only high 300-meters intervals were used for the reconstruction. Temperature anomalies obtained by the above mentioned technique (200-300 m sections were used as the base interval for each temperature record) are shown in Fig. 3. Most of anomalies both
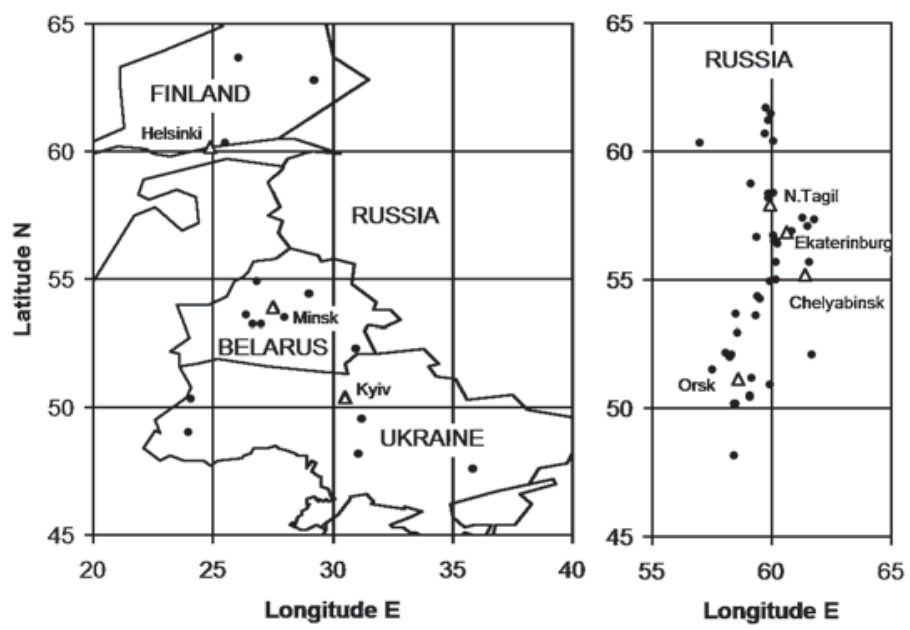

Fig. 2. Location of the boreholes in the Urals and Eastern Europe
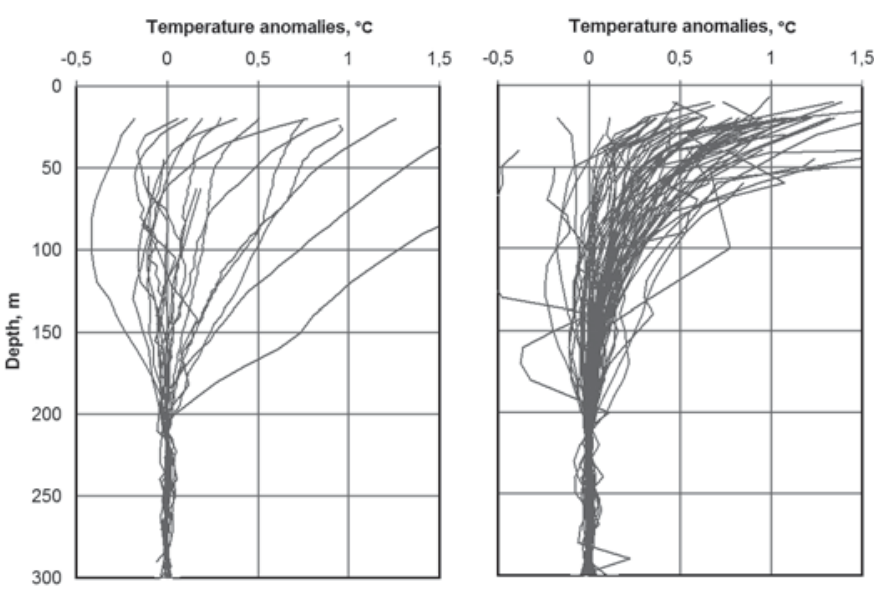

Fig. 3. Temperature anomalies for the Urals and Eastern Europe 
in the Urals and Eastern Europe reflect the warming process after the LIA.

Spatial analysis of paleoclimatic reconstructions requires that all investigated boreholes are identical in depth and that the reconstruction of GSTH is made by the same parameters with application the same method forall temperature logs [Beltrami,2003]. We used an equal thermal diffusivity of rocks = $=10^{-6} \mathrm{~m}^{2} / \mathrm{sec}$ in all cases. The reconstructed
GSTH from the $18^{\text {th }}$ to $20^{\text {th }}$ centuries compared with the long time series of meteorological records (annual surface air temperatures) are presented in Fig. 4. We can see that GSTHs and meteorological trends have a similar form reflecting warming process.

The data for the $20^{\text {th }}$ century were excluded from further consideration. Firstly, the temperature histories have different duration in the $20^{\text {th }}$ century because of different
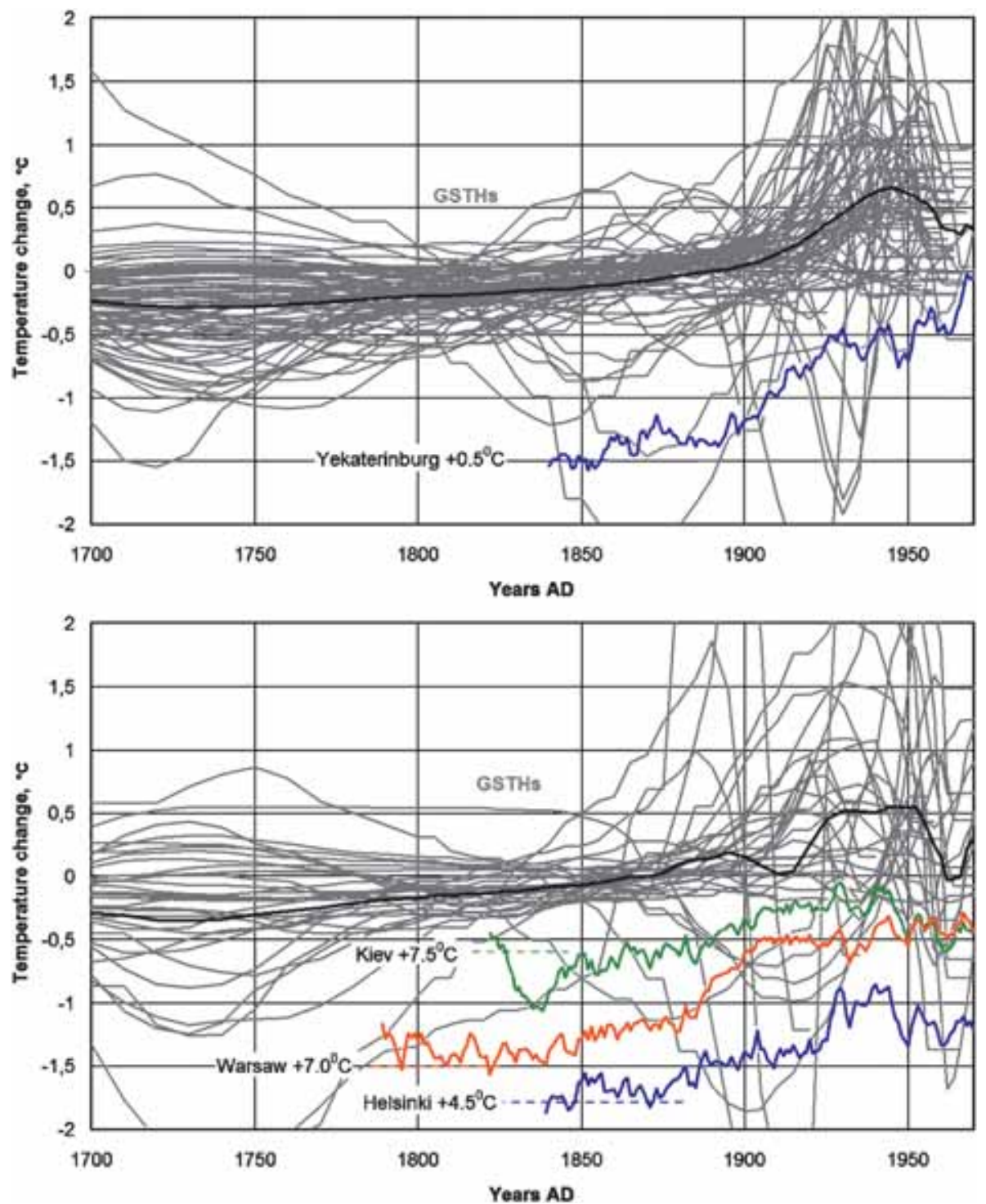

Fig. 4. Spaghetti diagram of the reconstructed GSTHs for the Urals (upper panel) and Eastern Europe (bottom panel). Each history is centered by its average temperature over the period from 1700 to 1960 (grey curves). Colour curves - long time series of air temperature records in the nearest cites averaged by 11-years running windows 

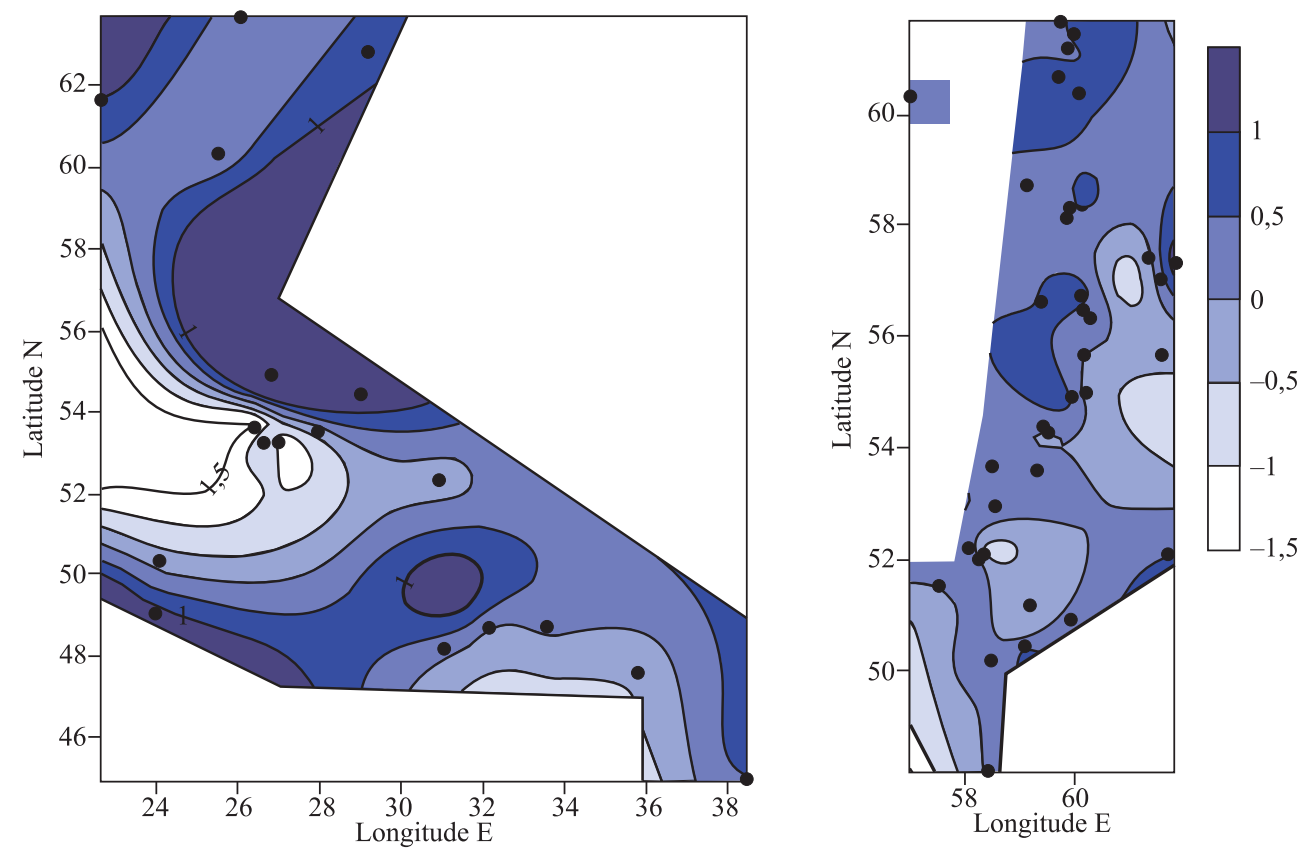

Fig. 5. Spatial distribution of the amplitudes of ground surface temperature changes $\left({ }^{\circ} \mathrm{C}\right)$ in the Urals and Eastern Europe from 1700 to 1900

positions of the temperature profiles' high points. Also, Fig. 4 displays extremely large variability of the reconstructing temperatures in $20^{\text {th }}$ century which is, probably, due to natural reasons as well as to the influence of nonclimatic factors. Spatial analysis of such data doesn't reveal any spatial regularities, whose detection is our main investigative objective. The second reason for the exclusion of the $20^{\text {th }}$ century data from consideration is that the influence of thermal effusivities contrast in the uppermost active layer is most significant for the data of such recent period [Kotlovanova, 2011]. The research results related to the period from 1700 to 1900 are presented below.

Spatial distribution of the amplitudes of ground surface temperature changes in the Urals and Eastern Europe from 1700 to 1900 is shown in Fig. 5.

As follows from Fig. 5, the amplitudes of temperature changes vary considerably for different regions. Positive values of the amplitudes correspond to climate warming in north and northwest Urals as well as to north, south, and all eastern area of Eastern Europe. The negative amplitudes trail along the south- west - northeast lines along the Urals and they are common to the central part of the European territory. Such spatial heterogeneity of the amplitudes may be due to the air masses motion transferring large masses of warm and cold air. Primary warming in the northern territory may be associated with the influence of the North Atlantic region. The role of local events for the climatic system is essential. These local phenomena may be connected with a land human invasion, landscape changes, and other anthropogenic activity. Atmospheric precipitation may be another factor that determines such distribution of the amplitudes. Snow cover increases soil temperature and summer precipitation decreases it. Spatial heterogeneity of precipitation is well above spatial heterogeneity of air temperature. These local phenomena of climatic system of the regions under study may be caused by the effect of precipitation quantity adjustment. Therefore, the positive amplitudes may be due to the snow cover height increase in these regions.

To compare the GSTHs shapes, we standardized them. Each GSTH was centered by its average value over the period from 

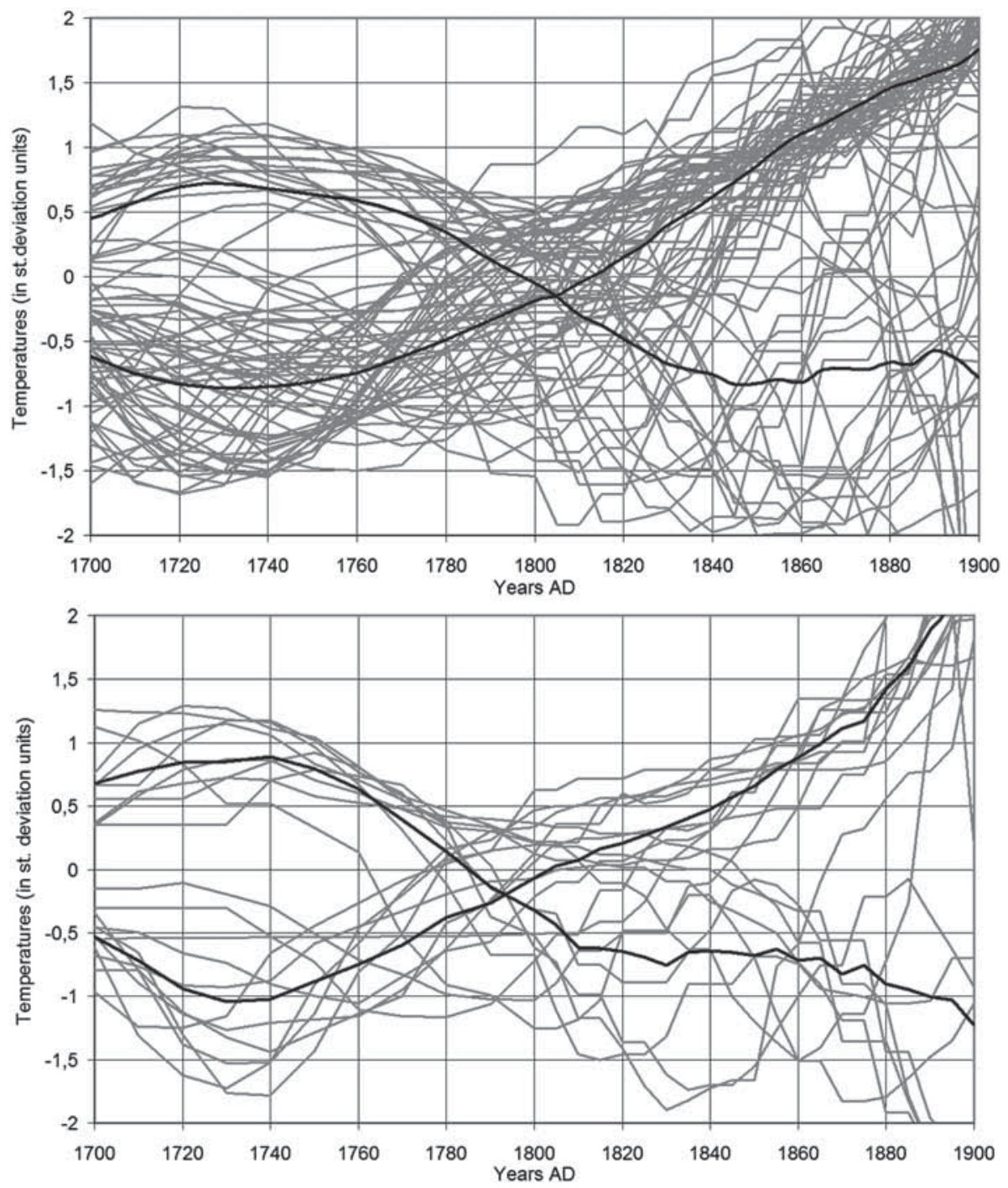

Fig. 6. Spaghetti diagram of the standardized temperature curves for the Urals (upper panel) and Eastern Europe (bottom panel). Black curves - mean GSTHs for two clusters

1700 to 1900 and was divided by the standard deviation. The standardized temperature curves are presented in Fig. 6.

Cluster analysis of the standardized temperature curves reveals at least two types of histories: with early (1720-1760) and late (after 1820) warming start date both in the Urals and Europe.

The spatial distribution of a warming start date is shown in Fig. 7. The earliest warming start is typical for the north-western part of the Urals and the northern regions of
Europe. Later, climatic changes occurred in the central European area.

\section{CONCLUSION}

Investigation of the spatial feature of climatic changes helps understanding the origin of the Earth's climatic cycles, the latest of which was the LIA. There is some evidence of these changes. A proxy paleoclimate evidence (tree-ring data, pollen evidences) is based on the assumption that correlations between measured quantities and estimated climate elements in the past are close to their present- 

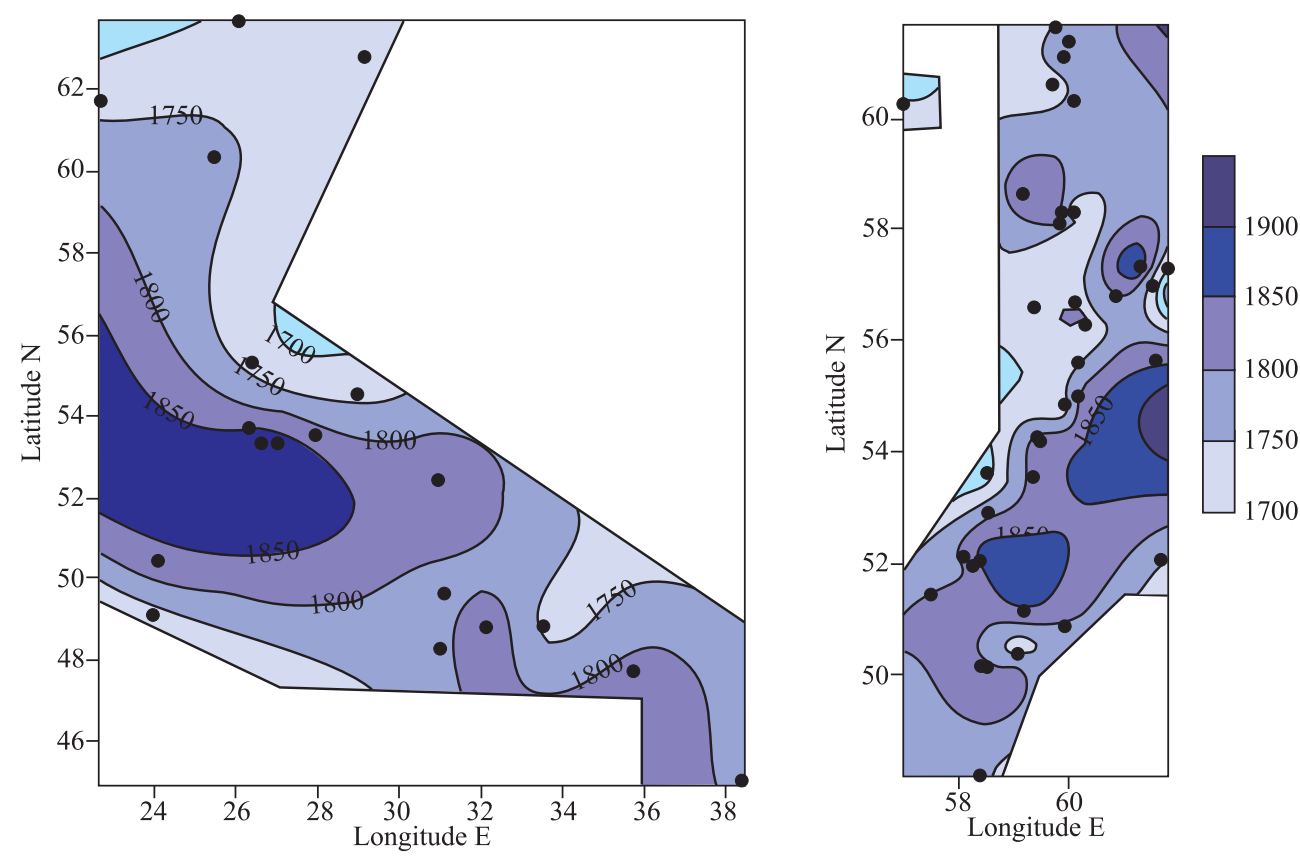

Fig. 7. Spatial distributions of a warming start date (year AD) in the Urals and Eastern Europe from the 18th to 20th centuries

day means. The geothermal method doesn't require establishing correlation links between measured and reconstructed temperatures.

Our investigation reveals distinct spatial patterns of ground surface temperature changes in the Urals and Eastern Europe in the $18^{\text {th }}-19^{\text {th }}$ centuries. There were two trends of temperature changes (warming and cooling), as manifested in both regions. The spatial distribution of the warming start dates in Eastern Europe agrees, in general, with GSTH reconstructions of Majorowicz (2010). An early warming start in the $18^{\text {th }}$ century in the northern parts of the Urals and Europe was recently found by Ljungqvist et al (2012) from multiproxy data analysis (excluding borehole). Nevertheless, the influence of non-climatic factors on the geothermal reconstruction is obvious. Deforestation and land use may change ground surface temperature by several degrees while air temperature remains stable. Deviation of the real geological structure from the model of homogeneous medium also disturbs the underground temperature field. However, most of these factors have a low spatial correlation and appear as a mosaic component of the spatial pattern. The most effective way to suppress a non-climatic influence on the regional reconstructions is integration of the geothermal method with other proxies.

\section{ACKNOWLEDGEMENTS}

This work was supported by the RFFI grant 10-05-00067. We would like to thank two anonymous reviewers for their helpful comments.

\section{REFERENCES}

1. Beltrami H., Gosselin C., and Mareschal J.C. (2003) Ground surface temperatures in Canada: Spatial and temporal variability. Geophys. Res. Lett., 30 (10), 1499, doi:10.1029/2003L017144.

2. Beltrami H., and Mareshal J.-C. (1991) Recent warming in eastern Canada inferred from geothermal measurements. Geophys. Res. Lett., vol. 18, N4:605-608. 
3. Carslaw H.S., Jaeger J.C. (1959) Conduction of Heat in Solids. $2^{\text {nd }}$ edn. Oxford Univ. Press, New York, 510 pp.

4. Cermak V., Bodri L., Safanda J. (1992) Underground temperature fields and changing climate: evidence from Cuba. Paleogeogr. Paleoclim. Paleoecol., 97, p. 325-327.

5. Demezhko D.Yu., and Golovanova I.V. (2007) Climatic changes in the Urals over the past millennium - an analysis of geothermal and meteorological data. Climate of the Past, 3 , p. 237-242 (http://222.clim-past.net/3/237/2007/cp-3-237-2007.html).

6. Demezhko D.Yu., and Shchapov V.A. (2001) 80,000 years ground surface temperature history inferred from the temperature-depth log measured in the superdeep hole SG-4 (the Urals, Russia). Glob. and Planet. Change, 29, p. 219-230.

7. Huang, S. and Pollack, H.N. (1998) Global Borehole Temperature Database for Climate Reconstruction. IGBP PAGES/World Data Center-A for Paleoclimatology Data Contribution Series \#1998-044. NOAA/NGDC Paleoclimatology Program, Boulder CO, USA. (http:// www.geo.Isa.umich.edu/climate/).

8. Kotlovanova A.A. (2011) Influence of thermal effusivity on propagation of temperature waves in subsurface. XII Ural youth scientific school on geophysics: Collection of scientific papers. - Perm, UB RAS, p. 119-122. (in Russian).

9. Lachenbruch A.H., and Marshall B.V. (1986) Changing climate: geothermal evidences from permafrost in the Alaskan Arctic. Science, 234, p. 689-696.

10. Ljungqvist F. C., Krusic P. J., Brattstrom G., and H. S. Sundqvist (2012) Northern Hemisphere temperature patterns in the last 12 centuries // Clim. Past, 8, p. 227-249.

11. Majorowicz J. (2010) The Climate of Europe in Recent Centuries in the Context of the Climate of Mid to High Latitude Northern Hemisphere from Borehole Temperature Logs / In: Przybylak, R.; Majorowicz, J.; Brázdil, R.; Kejan, M. (Eds.). The Polish Climate in the European Context: An Historical Overview. Springer Verlag, 1, p. 103-126, DOI: 10.1007/978-90-481-3167-9_4.
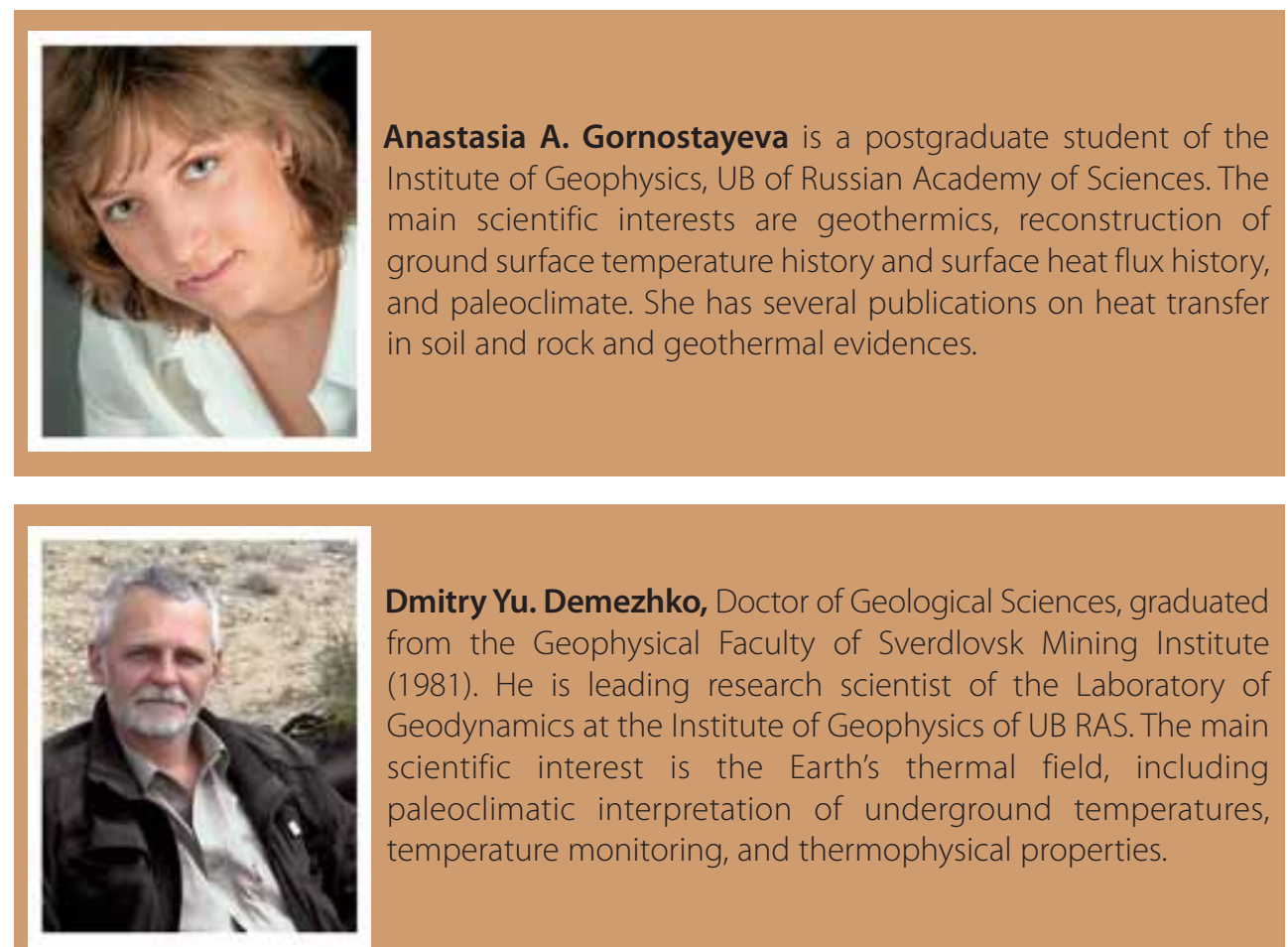

Dmitry Yu. Demezhko, Doctor of Geological Sciences, graduated from the Geophysical Faculty of Sverdlovsk Mining Institute (1981). He is leading research scientist of the Laboratory of Geodynamics at the Institute of Geophysics of UB RAS. The main scientific interest is the Earth's thermal field, including paleoclimatic interpretation of underground temperatures, temperature monitoring, and thermophysical properties. 